\title{
SARS-CoV-2 and its dynamic impact on emergency department preparedness and management
}

\author{
Sohil Pothiawala* \\ Department of Emergency Medicine, Woodlands Health Campus, Singapore
}

\author{
Received: 27 April 2020 \\ Accepted: 22 June 2020 \\ Published online: 29 June 2020 \\ *Corresponding author: Sohi \\ Pothiawala, Department of \\ Emergency Medicine, Woodlands \\ Health Campus, Singapore. \\ Email:drsohilpothiawala@yahoo. \\ com \\ Competing interests: None. \\ Funding information: None. \\ Citation: Pothiawala S. Journal of \\ Emergency Practice and Trauma \\ 2020; 6(2): 102-108. doi: 10.34172/ \\ jept.2020.25.
}

\begin{abstract}
Introduction: Emerging viral diseases (EVDs) pose a significant threat to public health. There have been a few viral epidemics in the last two decades. Coronavirus disease 2019 (COVID-19) is now a global pandemic.

Objective: To provide emergency physicians an update on severe acute respiratory syndrome coronavirus 2 (SARS-CoV-2) and its dynamic impact on the emergency department (ED) in terms of preparedness and management of patients presenting to ED with suspected COVID-19 disease.

Discussion: COVID-19 has a human-to-human transmission through close contact and even from asymptomatic carriers. Symptoms are similar to a viral respiratory illness. The disease is mild and self-limiting in most patients, but some develop severe illness like pneumonia, acute respiratory distress syndrome (ARDS) and multiorgan failure. Emergency physicians should prioritize early identification of these patients at risk, isolate them and after collaborating with relevant hospital and national authorities, develop clinical pathways to safely evaluate, manage and dispose patients with COVID-19. Various strategies for managing these patients in the ED are discussed. Disposition of these patients depends on symptoms and hemodynamic status and potential to self quarantine versus admission to an isolation facility.

Conclusion: This review provides an overview of patients presenting to the ED with suspected COVID-19 and its impact on the ED. It reiterates the fact that emergency physicians, in close collaboration with relevant hospital authorities, play a pivotal role during EVDs. It emphasizes the need for pandemic preparedness, enabling us to better manage such events in the future.
\end{abstract}

Keywords: SARS-CoV-2, COVID-19, Emergency department, Impact, Preparedness

\section{Introduction}

Emerging viral diseases (EVDs) pose a significant threat to public health. The last two decades have witnessed several viral epidemics, including SARS-CoV-1 in 2003, H1N1 influenza in 2009, MERS-CoV in 2012 and Ebola in 2014.

In December 2019, an unknown outbreak of several cases of pneumonia was identified in Wuhan City, Hubei province of China. It was thought to be associated with exposure in a seafood wholesale market there. The Chinese authorities isolated the virus on $7^{\text {th }}$ January 2020 and called it the novel coronavirus (2019-nCoV). It was then renamed as severe acute respiratory syndrome coronavirus 2 (SARSCoV-2), due to its clinical similarity to SARS. On $30^{\text {th }}$ January 2020, the World Health Organization (WHO) announced this outbreak as a "Public Health Emergency of International Concern" (PHEIC). On February 11, 2020, WHO announced that this disease would be termed
Coronavirus Disease 2019 (COVID-19). On March 11, 2020, WHO declared COVID-19 to be a global pandemic $(1,2)$. As of April 30, 2020, WHO has reported 3090445 cases of COVID-19 globally, with 217769 deaths (3).

The 2003 SARS outbreak and the 2014 Ebola outbreak have already highlighted the importance of emergency preparedness, redesigning the emergency departments (EDs) and hospital infrastructure and processes. They have highlighted the important role of the emergency physicians in the initial identification and management of these EVDs (4). This review describes the evolving literature on COVID-19 and its impact on the ED as well as explaining an approach to the initial management of patients with suspected COVID-19.

\section{Discussion}

SARS-CoV-2 is a single-stranded RNA virus, having crown-like spikes on its round surface when seen on an 
electron microscope (5). Like the previous coronaviruses, it is naturally hosted by bats, with some studies suggesting pangolin as an intermediate animal reservoir to infect humans. SARS-CoV-2 binds to the angiotensin converting enzyme II (ACE2) receptors in the human host, similar to SARS-CoV (6).

The majority of initial COVID-19 cases were identified in the residents of Hubei province, China or in people who had travelled to and from that region. Over the last few months, more and more cases reported across the world are secondary to human-to-human transmission.

The incubation period of COVID-19 ranges from 3-14 days, with a mean incubation period of 5.2 days (95\% CI 4.1-7.0) (7). The longest documented time to symptom evolvement is 12.5 days ( $95 \%$ CI, 9.2-18 days).

Experts believe that once a human is infected with SARS$\mathrm{CoV}-2$, there is human-to-human viral transmission through respiratory droplets generated by coughing and sneezing up to a distance of approximately 6 feet (8). Virus is also transmitted through fomites, with subsequent touching of the person's eyes, nose, or mouth. Patients who are more symptomatic have the highest risk of spreading the virus.

Feco-oral transmission of SARS-CoV-2 leads to gastrointestinal involvement infection, but more studies are needed to confirm this mode of transmission. Recent literature has also highlighted the importance of asymptomatic and pre-symptomatic virus shedding in the transmission of COVID-19, as it poses a big challenge in the identification of suspected cases as well as the prevention of local transmission (9-11).

In a study conducted in China, $86.6 \%$ of patients were $30-79$ years of age, with pediatric patients accounting only $2.1 \%$ of all cases. $80.9 \%$ of these cases were mild and $14.6 \%$ were severe or critical. The overall mortality rate was $2.3 \%$, with the highest mortality in patients older than 80 years (14.8\%) (12). Compared with the mortality rate of other recent epidemics, SARS (10\%), Ebola (50\%) and MERS$\mathrm{CoV}(34 \%)$, the average mortality rate for COVID-19 $(3.4 \%)$ is definitely lower.

Advanced age, chronic medical conditions such as chronic kidney disease, chronic pulmonary and cardiovascular disease, immunocompromised state and cancer are potential risk factors for severe COVID-19 (13) There are increasing reports of health care workers being exposed to the virus while caring for patients. This emphasizes the fact that emergency physicians as well as other ED staff must be cognizant of the risk of exposure at work and hence observe appropriate infection control guidelines stipulated by the Centers for Disease Control and Prevention (CDC) as well as their respective institutions (14).

Majority of COVID-19 cases present with an acute respiratory infection, the commonest symptoms being fever, dry cough and dyspnea. Patients may also present with vague generalized complaints like sore throat, sneezing, fatigue, lethargy and myalgia. Occasionally, some patients have gastrointestinal (GI) symptoms like diarrhea, vomiting, abdominal pain and anorexia. There have been anecdotal case reports of anosmia and dysgeusia as the only presenting complaints (13).

The lesser incidence of fever in SARS-CoV-2 infection, relative to SARS-CoV1 and MERS-CoV, raises concern regarding the use of fever detection as the only surveillance tool. The elderly or immunocompromised patients may present with atypical manifestations of COVID-19, and they may not spike a fever or demonstrate other typical symptoms that help clinch the diagnosis.

The list of differential diagnosis for COVID-19 can be huge, and should be individualized based on the patient's presenting symptoms as well as underlying co-morbidities. Influenza, viral illnesses like dengue, bacterial/aspiration pneumonia or other pulmonary diseases like pulmonary embolism should be considered in the appropriate setting. A detailed history including patient's travel history, history of contact with any positive COVID-19 patient and a detailed physical examination remain the key towards establishing an appropriate diagnosis.

Based on the clinical presentation, patients with COVID-19 are classified into mild, moderate, severe and critical category (15).

- Mild: fever, cough, sore throat, fatigue, malaise, vomiting, diarrhea, anosmia, but no radiological signs suggestive of pneumonia

- Moderate: fever, cough and shortness of breath along with radiological signs suggestive of pneumonia

- Severe: dyspnea, respiratory rate $\geq 30$ /minute, oxygen saturation $\leq 93 \%$ on room air, $\mathrm{PaO} 2 / \mathrm{FiO} 2$ ratio $<300$, and/or lung infiltrates $>50 \%$ of the lung field

- Critical: septic shock, acute respiratory distress syndrome (ARDS), multi organ dysfunction syndrome and coagulation dysfunction.

Patients with severe COVID-19 infection can develop complications like secondary bacterial infection, acute kidney injury, ventilator-associated pneumonia, acute coronary syndrome, ischemic stroke and encephalitis to name a few (16).

\section{General approach towards COVID-19 in the ED}

ED should adopt a standardized, multi-faceted approach for managing patients presenting with suspected EVD, including COVID-19. The primary focus should be on early identification of these at-risk patients, both in the pre-hospital as well as the hospital setting. The aim should be to isolate these patients and manage them accordingly, to avoid risk to other patients who are in the ED or the hospital.

While dealing with such outbreaks, there must be appropriate coordination and collaboration between $\mathrm{ED}$, relevant departments like Infectious Diseases and Intensive care Units, hospital management, Ministry of Health or other government agencies. Relevant messages, instructions and workflows and their updates must be 
disseminated regularly through a pre-determined channel. All positive or suspect EVD cases must be notified in a timely manner, so that an appropriate record can be kept at institutional as well as national level.

At the departmental and hospital level, there should be mechanisms in place to ensure adherence to appropriate infection control measures like hand washing, donning and doffing of personal protective equipment (PPE). Every staff must be adequately trained in this regard and must follow the appropriate steps. Contact tracing and syndromic surveillance can be better achieved by utilization of electronic health records. New technologies can be adopted for contact tracing of patients as well as healthcare staff who may have been involved in care of a positive COVID-19 patient, throughout the patient's journey at a healthcare institution. Clinicians must exercise caution and clinical judgment while reviewing results of the published literature, because their conclusion and suggestions are often based on studies carried out in a particular setting, and hence they may not be directly applicable to other settings.

\section{Managing suspected COVID-19 patients in the Pre- hospital setting}

Emergency medical service (EMS) department, in consultation with the Health Ministry should step up emergency preparedness measures to identify suspected COVID-19 patients and ensure safety of EMS personnel responding to them. EMS personnel arriving on-scene and providing direct patient care to these at risk patients should be geared in appropriate PPE based on the local health advisory. Ideally, they should be dressed in gown, gloves, N95 respirator, face shield or eye goggles. Aerosolgenerating procedure like nebulization should be avoided in the ambulance. Upon arrival at the ED, the patient should be transferred directly to the designated treatment area. The ambulance doors should be left open for proper air-circulation and the ambulance must be cleaned using approved disinfectant wipes. The paramedics should wear appropriate PPE (disposable gown, gloves, surgical mask, and face shield) while cleaning the ambulance (17).

\section{Managing COVID-19 patients in the ED}

Gaining insights from SARS epidemic in 2003, potentially infected patients in Singapore are managed with high vigilance, especially in these rapidly evolving conditions. Before entering the ED, all patients and accompanying person/visitors have to fill a rapid screening questionnaire on their travel history, symptoms of fever plus acute respiratory illness and history of contact with a suspicious case. Their body temperature is checked and documented. This pre-triage screening is done outside the ED entrance. The rationale is to identify a potentially suspected case as early as possible. Any patient with fever and a positive response to any question in the questionnaire is then managed at the designated isolation area of the
ED. Other entrances of the ED also need to be guarded, especially during this high risk period, to prevent at-risk patients from inadvertently entering the ED (18). Limit visitors entering the ED to only those who are essential (e.g. immediate family members or parent, guardian, or primary caregiver) and even they should be screened at the entry point with the screening questionnaire. As the disease becomes widespread to other countries in the world, including local transmission, modifying the screening questionnaire appropriately aids in identifying these potential cases. The ED as well as the hospital should monitor the daily attendance, and be prepared to redesign workflows of patient flow to best manage the pandemic.

Every suspected COVID-19 patient should be separated from another patient by at least 2 meters (6 feet). Both the patient as well as the accompanying person should wear a facemask for the entire duration of their consult till disposition. This will help reduce the risk of transmission to other surrounding patients in the ED. The isolation areas in ED have rooms with negative pressure ventilation. There should be ante-rooms to reduce the risk of transmission during opening and closing of doors, and they also serve as an area for health care workers to don/ doff the PPE before entering the isolation facility. The movement of staff in the isolation areas of the ED should be limited to those involved in patient care.

Healthcare workers should undergo appropriate PPE and hand hygiene training as per hospital guidelines, and these should be strictly followed before and after contact with every patient. A powered air purifying respirator (PAPR) must be used for patients requiring an aerosol-generating procedure like endotracheal intubation. As far as possible, basic radiological investigations should be catered for within the isolation area, limiting moving the patient to another location. If an investigation cannot be completed within the isolation facility and the patient needs to be transported to other areas of the ED or hospital, personnel involved in transport as well as those at the receiving location should wear appropriate PPE (19).

The Bed Management Unit of the hospital should also be aware of the various admission workflows, so they can appropriately allocate beds to cohort the group of patients, and thus avoid cross-contamination to other patients and staff. After appropriate disposition of the patient from the ED, disinfection and cleaning of the areas is crucial. Personnel involved in the cleaning process must follow the standard precautions and wear PPE, as the infectious particles may be present, although exact duration remains unclear (20).

(i) Laboratory and radiographic investigations in the ED The ED physician should perform certain basic laboratory and radiological investigations, including nasopharyngeal swabs that can aid in the diagnosis of the patient. These investigations should not be interpreted in isolation, but they should always be correlated with the clinical 
presentation of the patient.

\section{Hematological}

Leukopenia, especially lymphocytopenia, was seen in about $80 \%$ of the cases. Mild thrombocytopenia is also noted, similar to other viral illnesses. Neutrophil-tolymphocyte ratio (NLR) $\geq 3.13$ may serve as a marker for progression to severe illness in COVID-19 patients.

\section{Inflammatory markers}

COVID-19 is known to cause an inflammatory cascade, including coagulation disorders in the affected patient. Serum procalcitonin may be normal initially. There will be elevated levels of other inflammatory markers like C-reactive protein (CRP), lactate dehydrogenase ( $\mathrm{LDH}$ ), D-dimer, creatine kinase and erythrocyte sedimentation rate (ESR). As disease progresses, serum creatinine will be elevated in patients with acute kidney injury. Critically ill patients may demonstrate elevated levels of interleukin (IL)-6, IL-4, IL-10, and tumor necrosis factor (TNF)- $\alpha$, secondary to the underlying cytokine storm.

\section{Reverse transcription polymerase chain reaction}

Detecting the virus by genomic sequencing using reverse transcription polymerase chain reaction (RT-PCR) is the current confirmatory test for COVID-19. For RT-PCR in the ED, specimen collection via nasopharyngeal swab or sputum sample should be carried out wearing adequate PPE. Results of the RT-PCR may take a few hours up to 2 days, depending on the turnaround time of the laboratory. The specificity of RT-PCR is high, but its sensitivity can be low as about $70 \%$. The sensitivity may reduce further and the test may fail to detect the infection if the sample was not collected appropriately, or the patient was in the early stages of the disease with low viral load, or the test was carried out at a late stage of infection (21). The swab should be taken early at symptom onset, to obtain high virus concentrations (22). Symptomatic patients with a negative RT-PCR test usually do not have the disease (23). But a single negative RT-PCR should not be used to exclude the diagnosis, and the patient can be discharged with advice to self isolate. The RT-PCR should be repeated a few days later to confirm the presence or absence of the disease. Testing for alternative diagnoses like influenza may be considered for these patients (24).

\section{Radiology}

Plain chest $\mathrm{x}$-ray (CXR), point-of-care lung ultrasound and computed tomography (CT) of thorax can be performed on a case by case basis.

Although less sensitive than CT, CXR is the first line imaging modality. Findings are non-specific and CXR may be normal in early or mild disease. The most frequent findings are airspace opacities, described as consolidation or ground glass opacities (GGOs), often bilateral, and predominantly distributed in the peripheral and lower zones of the lung.

Lung ultrasound may be useful in the evaluation of critically ill COVID-19 patients. The findings are typically noted in multiple lung zones, as focal to diffuse multiple B-lines, irregular, thickened pleural line and pneumonic consolidation. Lung ultrasonography plays a role for rapid assessment of severity of COVID-19 pneumonia and it is safe, done at the bedside, repeatable, low cost and there is absence of radiation (25).

The primary CT changes that have been reported include bilateral, peripheral and basal ground-glass opacities (GGO), crazy paving appearance of GGOs, inter and intra-lobular septal thickening, air space consolidation and bronchovascular thickening in the lesion (26).

\section{(ii) Treatment}

Till date, no definite treatment exists for patients with COVID-19. Emergency physicians, infectious disease specialists, and health department/ministry should collaborate and development a clinical management pathway to safely evaluate, manage and dispose patients with COVID-19.

Close observation and early supportive care remains the mainstay of treatment. Most patients with COVID-19 do not require specific antiviral treatment except supportive care and can be managed symptomatically with close monitoring. About $15-20 \%$ of the patients progress to severe pneumonia and about 5\% require critical care. This subset of patients who progress to severe disease may benefit from early treatment with antiviral medications (27). This is from the small studies published till date, but definitive data from randomized controlled trials are still awaited. Intravenous antibiotics are recommended for patients who are critically ill or those with pneumonia on radiological investigations. Immunomodulator therapies (eg. IL-6 inhibitors) have also been proposed to limit the cytokine-mediated inflammation in critically ill patients with ARDS.

Breathless and hypoxic patients with a potential COVID-19 infection should be commenced on supplementary oxygen to maintain an oxygenation saturation of $90-96 \%$. Intubation and nebulization are considered high-risk aerosol-generating procedures. For patients in respiratory failure or ARDS requiring emergent intubation in the $\mathrm{ED}$, the following recommendations (28) need to be considered:

- Avoid administration of nebulized medication and prefer using MDI with spacer instead

- If oxygenation and ventilation support is needed, intubation is preferred instead of initial management with Non-Invasive Positive Pressure Ventilation (NIPPV) or High Flow Nasal Oxygenation (HFNO)

- Intubate only in a negative pressure room

- Restrict the members of an intubation team to a maximum of 3 people

- Personnel performing intubation should wear 
adequate PPE - at least N95 mask, goggles, face shield, gown, and gloves pulled over end of gown sleeves

- Use of PAPR is recommended during intubation, considering the risk of infection transmission of SARS-CoV-1 to healthcare workers while using only N95 respirators

- Most experienced available clinicians should intubate to maximize first attempt success

- Rapid sequence intubation (RSI) is the preferred method

- Avoid awake intubation, unless when absolutely indicated, because of the potential for coughing during application of topical anesthesia, leading to virus transmission

- Use high-dose neuromuscular blocking agents for faster and more complete apnea and no residual cough

- $\quad$ Preoxygenate the patient for at least 5 min with $100 \%$ oxygen before RSI. Use of NIPPV for preoxygenation is not recommended due to the increased risk of aerosolization

- Use video laryngoscope compared to direct laryngoscope to increase the distance between the operator and the patient

- Clinicians can use a screen instead of having to look in the patient's mouth and thus increase operator to patient distancing

- A high-efficiency particulate air filter should be attached to the expiratory limb of the mechanical ventilator

Post-intubation, the initial ventilator settings should be set using tidal volumes of $4-8 \mathrm{~mL} / \mathrm{kg}$ of predicted body weight, respiratory rate of $16-20$ breaths/min, PEEP of 5 $\mathrm{cm} \mathrm{H}_{2} \mathrm{O}$ and plateau pressures $<30 \mathrm{~cm} \mathrm{H}_{2} \mathrm{O}$.

Emergency physicians should aim to identify the early signs of septic shock to avoid deterioration. Judicious use of intravenous fluids, especially crystalloids, is recommended for controlled fluid resuscitation. The first choice of vasopressors for persistent shock despite fluid resuscitation should be norepinephrine, with a target to achieve mean arterial pressure of 60-65 mm $\mathrm{Hg}$. For patients with persistent hypotension despite norepinephrine, epinephrine or vasopressin infusion should be commenced, instead of dopamine. Dobutamine is recommended for patients having cardiac dysfunction on bedside echocardiography (28). The routine use of corticosteroids is not recommended, except in refractory shock states or documented hypocortisolism (27).

Regarding antiviral therapy, if lopinavir-ritonavir use is considered for patients with severe COVID-19, its use within 12 days of symptom onset is associated with earlier improvement of symptoms (hazard ratio 1.25; 95\% CI 1.77-2.05) (29). The use of remdesivir has shown potent antiviral activity against SARS-CoV-2 in in-vitro studies and some case reports. Till RCT results that are expected soon, it is recommended to use remdesivir under a trial/ study setting $(30,31)$.

There is no published study regarding the use of interferon beta-1B in SARS-CoV-2. A small French study to treat patients with hydroxychloroquine and azithromycin showed reduction of viral load at day 6, compared to the study group, but more robust data in needed (32). Other medications under study include immunomodulators tocilizumab (IL-6 inhibitor) for patients in cytokine storm and favipiravir.

Trials regarding the use of specific antiviral drugs for treating COVID-19 patients and vaccine to control the spread of the pandemic are underway. Till then, observation of universal health precautions, isolation of suspect and/or positive patients and close monitoring with supportive care form the cornerstone of treatment.

\section{(iii) Disposition}

Patients with mild symptoms and no significant comorbidities may be discharged from the ED after a nasopharyngeal swab and given symptomatic medications and advice to self-quarantine at home for 2 weeks. Emergency physicians should counsel these patients and give them a discharge advisory to return back to the ED for new or worsening respiratory symptoms and fever. Mechanism should be in place to inform them of the swab result, and if positive, they may be advised to continue monitoring their symptoms at home or they should be admitted to a designated quarantine facility, depending on national protocols. The relevant health department/ ministry should collaborate in the care of these patients and they can follow-up with surveillance, contact tracing and repeat testing of this group of patients and their family members.

Patients with moderate to severe symptoms, with or without underlying co-morbidities, especially those having pneumonia on CXR or hypoxic patients requiring supplemental oxygen, will require admission for further management and monitoring (33).

\section{Conclusion}

SARS-CoV-2 is an evolving pandemic, transiting from initially affecting those who travelled to an affected area to local community transmission. It is more infectious than SARS-CoV-1 and MERS-CoV. A vast majority of patients will recover after a mild, self-limiting disease, but some will progress to severe illness or develop life threatening complications, eventually leading to death. Emergency physicians, in close collaboration with relevant hospital authorities, play a pivotal role during these EVDs, and should promptly adopt the principles of emergency preparedness whilst maintaining a high index of suspicion. This will aid in early identification of suspected COVID-19 patients in the ED, and adopting isolation measures. This will help reduce the exposure to other patients and healthcare workers as well as improve patient outcomes. 
The key to effective management of these outbreaks is collaboration and a consolidated strategy across the healthcare sector. At the hospital level, there must be continuity of care between ED and inpatient care. However, the pandemic preparedness must extend beyond the healthcare sector, involving the public as well as relevant partners at the national level. Regular conduct of disaster and pandemic drills to test our preparedness will play an important role and provide us an opportunity to identify our gaps and address them.

Let us learn from this pandemic, emerge from it, commit to evolving new strategies to optimally face such threats in the future.

\section{Authors' contributions}

SP conceived the idea for the manuscript and also contributed to the writing and editing of the review.

\section{Ethical Issues}

Not applicable.

\section{References}

1. World Health Organization [Internet]. Statement on the second meeting of the International Health Regulations (2005) Emergency Committee regarding the outbreak of novel coronavirus (2019-nCoV). [cited 2020 Apr 10]. Available from: https://www.who.int/news-room/ detail/30-01-2020-statement-on-the-second-meeting-ofthe-international-health-regulations-(2005)-emergencycommittee-regarding-the-outbreak-of-novel-coronavirus(2019-ncov).

2. World Health Organization [Internet]. WHO DirectorGeneral's opening remarks at the media briefing on COVID-19 - 11 March 2020. [cited 2020 Apr 10]. Available from: https://www.who.int/dg/speeches/detail/ who-director-general-s-opening-remarks-at-the-mediabriefing-on-covid-19-11-march-2020.

3. World Health Organization [Internet]. Coronavirus Disease 2019 (COVID-19) Situation Report - 101. 30th April 2020. [cited 2020 May 1]. Available from: https://www.who.int/docs/default-source/coronaviruse/ situation-reports/20200430-sitrep-101-covid-19. pdf?sfvrsn=2ba4e093_2.

4. Molinari NA, LeBlanc TT, Stephens W. The impact of a case of Ebola virus disease on Emergency Department visits in Metropolitan Dallas-Fort Worth, TX, July, 2013-July, 2015: An interrupted time series analysis. PLOS Currents Outbreaks. 2018 Mar 20. Edition 1. doi: 10.1371/currents. outbreaks.e62bdea371ef5454d56f71fe217aead0.

5. Centers for Disease Control and Prevention [Internet]. Human Coronavirus Types. [cited 2020 Apr 10]. Available from: https://www.cdc.gov/coronavirus/types.html.

6. Wong MC, Cregeen SJ, Ajami NJ, et al. Evidence of recombination in coronaviruses implicating pangolin origins of nCoV-2019. bioRxiv 2020. doi: 10.1101/2020.02.07.939207.

7. Li Q, Guan X, Wu P, Wang X, Zhou L, Tong Y, et al. Early transmission dynamics in Wuhan, China, of novel coronavirus-infected pneumonia. N Engl J Med 2020; 382(13):1199-207. doi: 10.1056/NEJMoa2001316.

8. Centers for Disease Control and Prevention [Internet]. 2019 Novel Coronavirus (2019-nCoV) transmission. [cited 2020 Apr 10]. Available from: https://www.cdc.gov/ coronavirus/2019-ncov/about/transmission.html.

9. Chan JF, Yuan S, Kok KH, To KK, Chu H, Yang J, et al. A familial cluster of pneumonia associated with the 2019 novel coronavirus indicating person-to-person transmission: a study of a family cluster. Lancet 2020; 395(10223): 514-23. doi: 10.1016/S0140-6736(20)30154-9.

10. Bai Y, Yao L, Wei T, Tian F, Jin DY, Chen L, et al. Presumed asymptomatic carrier transmission of COVID-19. JAMA 2020; 323(14): 1406-7. https://doi.org/10.1001/ jama.2020.1585

11. Yuen KS, Ye ZW, Fung SY, Chan CP, Jin DY. SARS-CoV-2 and COVID-19: The most important research questions. Cell Biosci 2020; 10: 40. doi: 10.1186/s13578-020-00404-4.

12. Chavez S, Long B, Koyfman A, Liang SY. Coronavirus Disease (COVID-19): A primer for emergency physicians. Am J Emerg Me 2020; S0735-6757(20)30178-9. doi: 10.1016/j.ajem.2020.03.036.

13. Centers for Disease Control and Prevention [Internet]. 2019 Novel Coronavirus (2019-nCoV) Clinical care. [cited 2020 Apr 10]. Available from: https://www.cdc. gov/coronavirus/2019-ncov/hcp/clinical-guidancemanagement-patients.html.

14. Centers for Disease Control and Prevention [Internet]. Interim U.S. Guidance for Risk Assessment and Public Health Management of Healthcare Personnel with Potential Exposure in a Healthcare Setting to Patients with Coronavirus Disease (COVID-19). [cited 2020 Apr 12]. Available from: https://www.cdc.gov/coronavirus/2019ncov/hcp/guidance-risk-assesment-hcp.html.

15. World Health Organization [Internet]. Director-General's remarks at the media briefing on COVID-2019 outbreak on17 February. [cited 2020 Apr 12]. Available from: https:// www.who.int/dg/speeches/detail/who-director-general-sremarks-at-the-media-briefing-on-covid-2019-outbreakon-17-february-2020.

16. Huang C, Wang Y, Li X, Ren L, Zhao J, Hu Y, et al. Clinical features of patients infected with 2019 novelcoronavirus in Wuhan, China. Lancet 2020; 395(10223): 497-506. doi: 10.1016/S0140-6736(20)30183-5.

17. Centers for Disease Control and Prevention [Internet]. Coronavirus Disease 2019 (COVID-19) Interim Guidance for Emergency Medical Services (EMS) Systems and 911 Public Safety Answering Points (PSAPs) for COVID-19 in the United States. [cited 2020 Apr 10]. Available from: https://www.cdc.gov/coronavirus/2019-ncov/hcp/ guidance-for-ems.html.

18. Lateef F. Hospital design for better infection control. J Emerg Trauma Shock 2009; 2: 175-9.

19. Centers for Disease Control and Prevention [Internet]. 2019 Novel Coronavirus (2019-nCoV) healthcare infection prevention and control FAQs for COVID-19. [cited 2020 Apr 12]. Available from: https://www.cdc.gov/ coronavirus/2019-ncov/hcp/infection-prevention-controlfaq.html.

20. Centers for Disease Control and Prevention [Internet]. 
Coronavirus Disease 2019 (COVID-19) Interim Infection Prevention and Control Recommendations. [cited 2020 Apr 12]. Available from: https://www.cdc. gov/coronavirus/2019-ncov/infection-control/controlrecommendations.html.

21. Guo L, Ren L, Yang S, Xiao M, Chang D, Yang F, et al. Profiling early humoral response to diagnose novel coronavirus disease (COVID-19). Clin Infect Dis 2020; ciaa310. doi: 10.1093/cid/ciaa310.

22. Gao X, Zhou H, Wu C, Xiao Y, Ren L, Paranhos-Baccalà G, Guo L, Wang J. Antibody against nucleocapsid protein predicts susceptibility to human coronavirus infection. J Infect 2015; 71(5): 599-602. doi: 10.1016/j.jinf.2015.07.002.

23. Centers for Disease Control and Prevention [Internet]. 2019 Novel Coronavirus (2019-nCoV) frequently asked questions and answers. [cited $2020 \mathrm{Apr} 10$ ]. Available from: https://www.cdc.gov/coronavirus/2019-ncov/faq.html.

24. Centers for Disease Control and Prevention [Internet]. 2019 Novel Coronavirus (2019-nCoV) evaluating and reporting PUI. [cited 2020 Apr 10]. Available from: https://www.cdc. gov/coronavirus/2019-ncov/hcp/clinical-criteria.html.

25. Peng QY, Wang XT, Zhang LN, Chinese Critical Care Ultrasound Study Group (CCUSG). Findings of lung ultrasonography of novel corona virus pneumonia during the 2019-2020 epidemic. Intensive Care Med 2020; 46(5): 849-850. doi: 10.1007/s00134-020-05996-6.

26. Singh A, Shaikh A, Singh R, Singh AK. COVID-19: From bench to bed side. Diabetes Metab Syndr 2020;14(4): 277281. doi: 10.1016/j.dsx.2020.04.011.

27. National Center for Infectious Diseases Singapore. Interim Treatment Guidelines for COVID-19. Version 1.0, 20202
Apr 2.

28. Alhazzani W, Møller MH, Arabi YM, Loeb M, Gong MN, Fan E, Oczkowski S, et al. Surviving Sepsis Campaign: guidelines on the management of critically ill adults with Coronavirus Disease 2019 (COVID-19). Intensive Care Med 2020; 46(5): 854-887. doi: 10.1007/s00134-020-060225.

29. Cao B, Wang Y, Wen D, Liu W, Wang J, Fan G, et al. A Trial of Lopinavir-Ritonavir in Adults Hospitalized with Severe Covid-19. N Engl J Med 2020;382(19):1787-99. doi: 10.1056/NEJMoa2001282

30. Wang $\mathrm{M}$, Cao R, Zhang L, Yang X, Liu J, Xu M, et al. Remdesivir and chloroquine effectively inhibit the recently emerged novel coronavirus (2019-nCoV) in vitro. Cell Res 2020; 30(3): 269-271. doi: 10.1038/s41422-020-0282-0.

31. Sanville B, Corbett R, Pidcock W, Hardin K, Sebat C, Nguyen MV, et al. A community transmitted case of severe acute respiratory distress syndrome due to SARS CoV2 in the United States. Clin Infect Dis 2020:ciaa347. doi: $10.1093 / \mathrm{cid} / \mathrm{ciaa} 347$.

32. Chen Z, Hu J, Zhang Z, Jiang S, Han S, Yan D, et al. Efficacy of hydroxychloroquine in patients with COVID-19: results of a randomized clinical trial. Medrxiv; 2020. https://doi.or $\mathrm{g} / 10.1101 / 2020.03 .22 .20040758$.

33. World Health Organization [Internet]. Home care for patients with suspected novel coronavirus $(\mathrm{nCoV})$ infection presenting with mild symptoms and management of contacts. [cited 2020 Apr 12]. Available from: https://www. who.int/publications-detail/home-care-for-patients-withsuspected-novel-coronavirus-(ncov)-infection-presentingwith-mild-symptoms-and-management-of-contacts. 